\title{
Controlled Quantum Teleportation Schemes Using Generalized Bell Bases
}

\author{
Xie $\mathrm{Wu}^{1, *}$, Ouyang Shan ${ }^{1,2}$ and Xiao Hailin ${ }^{2}$ \\ ${ }^{1}$ School of Electronic Engineering, Xidian University, Xi'an, 710071, P.R. China \\ ${ }^{2}$ School of Information and Communication, Guilin University of Electronic Technology, Guilin, 541004, P.R. China
}

\begin{abstract}
In view of the problem that there is only one typical Bell basis for QT (Quantum Teleportation), three new GBBs (Generalized Bell Bases) are obtained by transforming unitarily the Hadamard gates with the methods of Pauli gates in the quantum circuit of the traditional Bell basis, and four Bell bases have an identical general formula. Three controlled-QT schemes are proposed based on these GBBs. The changed processes of quantum entangled states during QT in these schemes are derived by using the similar method of the traditional Bell basis. The result analyses of quantum state collapses of controlled-QT show that more two-qubit quantum entanglement resources of Bell states can be available. These schemes can be considered as the beneficial supplements for current QT work with GBS (Generalized Bell State).
\end{abstract}

Keywords: Generalized bell bases, quantum communication, quantum entanglement, quantum information processing, quantum teleportation.

\section{INTRODUCTION}

Quantum teleportation (QT) is an important component of quantum information science as a novel quantum communication approach. It was originally presented by Bennett et al. [1] in 1993. Later, Bouwmeester et al. implemented the first QT experiment in 1997 [2], which attracted much attention from diverse countries. With this technique developing [3-7], QT has been widely applied in such fields as quantum key distribution [8, 9], quantum repeater communication $[9,10]$, quantum communication network [9, 11], etc, which make it occupy a significant position in QIP (quantum information processing). Since QT is closely related to Bell states, many researchers have generalized the traditional EPR (Einstein-Podorrsky-Rosen) States (i.e. Bell states) to investigate QT in deep, and have gained a lot of achievements which can be divided into the continuous-variable QT and discrete-variable QT. For the former, Hu et al. [12] put forward the QT schemes with the generalized EPR States based on Vaidman's work [13]. The fidelity of EPR States relies on the squeezing degrees of entanglement and the reflection coefficient of the beam splitter, and there is much work to do to realize the schemes for the instrument and environment reasons. In fact, most researchers dealt with the latter. For instance, Bennett et al. [1] extended the singlet state to $N$-state with a general explicit expression. Hence, the limits of two qubits for Bell states were broken through. The original general formulae of the multi-qubit GBS (Generalized Bell State) have been provided, which have promoted greatly the developments of GBS-QT [14-17]. In 2005, Rigolin [18] introduced 16 maximally entangled GBS (G states), and then Cao et al. [19] defined 16 non-maximally entanglement GBS (also called G states) based on Rigolin's work [18] in 2010. By applying these $\mathrm{G}$ states, they expanded the traditional Bell states from two qubits to four qubits to teleport perfectly unknown two-qubit quantum state. The methods of unilateral coefficients were employed to change the Bell states to generalize the protocols of teleportation [20-23]. Similarly, the approaches of double coefficients were adopted to generalize the Bell states for quantum teleportation [14, 24]. In 2012, Tanaka et al. [25] utilized the method of Latin square to construct a new kind of GBSs, and they obtained two-level and m-level perfect teleportation schemes. These GBSs contain Bennett's generalizations [1], yet Tanaka's teleportation schemes have the condition of nonmaximal entangled state.

These QT schemes with GBS above enrich significantly the theoretical researches of quantum communication, and the unknown quantum states of two or more qubits can be teleported, which provides many valuable approaches for the QIP applications.

However, most of current work has mainly focused on the GBS-QT schemes from two qubits to multi-qubits. The dimensions of Hilbert spaces have been expanded higher and higher. A new problem has occurred that the traditional Bell basis is single, and how to generalize this basis to teleport with EPR pairs only in four-dimensional space is needed to research. Unfortunately, whether there are other similar Bell bases in four-dimensional Hilbert space or not is still unclear. Moreover, these QT schemes lack the quantum circuits to generate GBS, which also brings some difficulties for the implementation of QT experiments. 
In this paper, motivated by the GBSs-QT schemes [1425], three novel Hadamard gates are introduced using Pauli gates to derive theoretically three new generalized Bell bases (GBBs) from the quantum circuits of the typical Bell basis, and three controlled QT schemes with GBBs are proposed as the beneficial supplements for current GBS-QT work.

\section{QT SCHEMES USING GBBS}

To describe more I/O (Input/Output) rules of Bell bases, four Bell states $\left(\left|\ddot{O}^{+}\right\rangle,\left|\varnothing^{+}\right\rangle,\left|\ddot{O}^{-}\right\rangle,\left|\varnothing^{-}\right\rangle\right)$of the traditional Bell basis [1] are described again as $\left|\beta_{i}\right\rangle$ ( $i=00,01,10,11$ ) respectively:

$$
\begin{aligned}
& \left|\beta_{00}\right\rangle=\left|\ddot{O}^{+}\right\rangle=(|00\rangle+|11\rangle) / \sqrt{2} \\
& \left|\beta_{01}\right\rangle=\left|\varnothing^{+}\right\rangle=(|01\rangle+|10\rangle) / \sqrt{2} \\
& \left|\beta_{10}\right\rangle=\left|\ddot{O}^{-}\right\rangle=(|00\rangle-|11\rangle) / \sqrt{2} \\
& \left|\beta_{11}\right\rangle=\left|\varnothing^{-}\right\rangle=(|01\rangle-|10\rangle) / \sqrt{2}
\end{aligned}
$$

The quantum circuit of the traditional Bell basis is shown as Fig. (1), and there exist a CNOT (Controlled-NOT) gate as Eq. (5) and a Hadamard gate as Eq. (6).

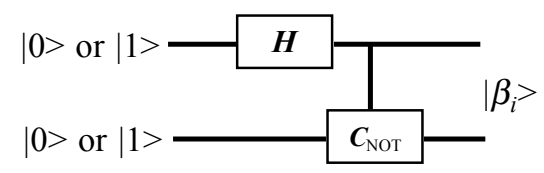

Fig. (1). Quantum circuit of the traditional Bell basis.

$$
\begin{aligned}
& \boldsymbol{C}_{\mathrm{NOT}}=\left[\begin{array}{llll}
1 & 0 & 0 & 0 \\
0 & 1 & 0 & 0 \\
0 & 0 & 0 & 1 \\
0 & 0 & 1 & 0
\end{array}\right] \\
& \boldsymbol{H}=\frac{1}{\sqrt{2}}\left[\begin{array}{cc}
1 & 1 \\
1 & -1
\end{array}\right]
\end{aligned}
$$

\subsection{Generalizations of the traditional Bell basis}

To measure quantum entangled states with more Bell bases in teleportation schemes, the Hadamard gate in Fig. (1) is transformed unitarily with three Pauli gates as $\boldsymbol{X}$ (i. e. $\left.\sigma_{x}\right),-\mathrm{j} \boldsymbol{Y}\left(\right.$ i. e. $\left.-\mathrm{j} \sigma_{y}\right), \boldsymbol{Z}$ (i. e. $\sigma_{z}$ ) [26]:

$$
\begin{aligned}
& \boldsymbol{H}_{1}=-\mathrm{j} \boldsymbol{Y H}=\frac{1}{\sqrt{2}}\left[\begin{array}{cc}
-1 & 1 \\
1 & 1
\end{array}\right] \\
& \boldsymbol{H}_{2}=\boldsymbol{X} \boldsymbol{H}=\frac{1}{\sqrt{2}}\left[\begin{array}{cc}
1 & -1 \\
1 & 1
\end{array}\right] \\
& \boldsymbol{H}_{3}=\boldsymbol{Z} \boldsymbol{H}=\frac{1}{\sqrt{2}}\left[\begin{array}{cc}
1 & 1 \\
-1 & 1
\end{array}\right]
\end{aligned}
$$

where $\mathrm{j}=\sqrt{-1}$.

$\boldsymbol{H}_{l} \boldsymbol{H}_{l}^{\dagger}(l=1,2,3)$ is an identity gate $(\boldsymbol{I}) . \dagger$ is the conjugate transpose. So, $\boldsymbol{H}_{l}$ can be considered as the generalizations of Hadamard gate for the similar forms and functions, and $\boldsymbol{H}_{1}, \boldsymbol{H}_{2}$ and $\boldsymbol{H}_{3}$ are called as the first, the second and the third Hadamard gates separately, while Eq. (6) is named as the fourth Hadamard gate $\left(\boldsymbol{H}_{4}\right)$. These new Hadamard gates are a little different from that of Ref. [17].

Now, the new Bell bases can be deduced with $\boldsymbol{H}_{l}$ instead of $\boldsymbol{H}$ in Fig. (1). If $l=1$, then the outputs $\left|\beta_{i}\right\rangle$ with $\boldsymbol{C}_{\text {NOT }}$ and the inputs of $|0\rangle$ (or $|1\rangle)$ are

$$
\begin{array}{ll}
\left|\beta_{00}\right\rangle=-(+|00\rangle & |11\rangle) / \sqrt{2} \\
\left|\beta_{01}\right\rangle=-(+01\rangle & |10\rangle) / \sqrt{2} \\
\left|\beta_{10}\right\rangle=+|00\rangle & |11\rangle) / \sqrt{2} \\
\left|\beta_{11}\right\rangle=+|01\rangle & |10\rangle) / \sqrt{2}
\end{array}
$$

From the Eqs. (10-13), the following formula of quantum entangled states holds.

$\left\langle\beta_{m} \mid \beta_{n}\right\rangle=\left\{\begin{array}{l}1, m=n \\ 0, m \neq n\end{array}\right.$

where $\langle\cdot \mid \cdot\rangle$ represents the inner product of quantum states. $m, n=00,01,10,11$.

Therefore, Eqs. (10-13) are orthogonal and normalized as well as Eqs. (1-4). These four maximally entangled states compose a new orthonormal basis which is generalized from the quantum circuit of the traditional Bell basis in Fig. (1). Here, Eqs. (10-13) are called as the first generalized Bell basis $\left(\boldsymbol{B}_{1}\right)$, while the traditional Bell basis are referred to as the fourth Bell basis $\left(\boldsymbol{B}_{4}\right)$ by Eq. (6).

Similarly, if $\boldsymbol{H}_{2}$ is substituted for $\boldsymbol{H}$ using the approach of $\boldsymbol{B}_{1}$, then four maximally entangled states can also be attained to form an orthonormal basis with the similar expression of $\boldsymbol{B}_{4}$, and it is named as the second generalized Bell basis $\left(\boldsymbol{B}_{2}\right)$. The Bell states of $\boldsymbol{B}_{2}$ with the same inputs as $\boldsymbol{B}_{4}$ are

$$
\begin{aligned}
& \left.\left|\beta_{00}\right\rangle=+|00\rangle \quad|11\rangle\right) / \sqrt{2} \\
& \left|\beta_{01}\right\rangle=+(|01\rangle \quad|10\rangle) / \sqrt{2} \\
& \left|\beta_{10}\right\rangle=-(+|00\rangle \quad|11\rangle) / \sqrt{2} \\
& \left|\beta_{11}\right\rangle=-(+|01\rangle \quad|10\rangle) / \sqrt{2}
\end{aligned}
$$

When $\boldsymbol{H}_{3}$ is connected instead of $\boldsymbol{H}$ in Fig. 1, an orthonormal basis can be achieved from $\boldsymbol{B}_{4}$ as well as $\boldsymbol{B}_{1}$ and $\boldsymbol{B}_{2}$, and it is designated as the third generalized Bell basis $\left(\boldsymbol{B}_{3}\right)$ with four Bell states:

$$
\begin{aligned}
& \left|\beta_{00}\right\rangle=-(|00\rangle \quad|11\rangle) / \sqrt{2} \\
& \left|\beta_{01}\right\rangle=-(|01\rangle \quad|10\rangle) / \sqrt{2}
\end{aligned}
$$




$$
\begin{array}{ll}
\left|\beta_{10}\right\rangle=+|00\rangle & |11\rangle) / \sqrt{2} \\
\left|\beta_{11}\right\rangle=+|01\rangle & |10\rangle) / \sqrt{2}
\end{array}
$$

\subsection{Equivalent Relationship of Unitary Transform Among Four Bell Bases}

According to quantum mechanics, the quantum collapse results of quantum states are different if different bases are chosen to measure quantum entanglement states. Therefore, it is necessary to find the transformation relations among four Bell bases with sixteen Bell states. Generally, the Bell states of four Bell bases can be represented as:

$\left.\boldsymbol{B}_{k}=\left\{\beta_{i}^{\boldsymbol{B}_{k}}\right\rangle \mid i=00,01,10,11\right\}$

where $k=1,2,3,4 \cdot\left|\beta_{i}^{\boldsymbol{B}_{k}}\right\rangle$ is a Bell state of $\boldsymbol{B}_{k}$.

The quantum gates to transform between $\boldsymbol{B}_{s}$ and $\boldsymbol{B}_{t}$ are denoted as $\boldsymbol{B}_{s t}$ and $\boldsymbol{B}_{t s}$, where $s, t \in\{1,2,3,4\}$. The linear relationships for these quantum gates to transform unitarily any Bell states between two Bell bases $\boldsymbol{B}_{s}$ and $\boldsymbol{B}_{t}$ satisfy the following equations:

$$
\begin{aligned}
& \left|\beta_{i}^{B_{s}}\right\rangle=\boldsymbol{B}_{s t}\left|\beta_{i}^{\boldsymbol{B}_{t}}\right\rangle \\
& \left|\beta_{i}^{\boldsymbol{B}_{t}}\right\rangle=\boldsymbol{B}_{t s}\left|\beta_{i}^{\boldsymbol{B}_{s}}\right\rangle
\end{aligned}
$$

where $i=00,01,10,11$.

By linear transformations, $\boldsymbol{B}_{s t}$ and $\boldsymbol{B}_{t s}$ are given by

$$
\begin{gathered}
\boldsymbol{B}_{12}=\boldsymbol{B}_{21}=\left[\begin{array}{cccc}
-1 & 0 & 0 & 0 \\
0 & -1 & 0 & 0 \\
0 & 0 & 1 & 0 \\
0 & 0 & 0 & 1
\end{array}\right] \\
\boldsymbol{B}_{13}=\boldsymbol{B}_{31}=\boldsymbol{B}_{24}=\boldsymbol{B}_{42}=\left[\begin{array}{cccc}
0 & 0 & 0 & 1 \\
0 & 0 & 1 & 0 \\
0 & 1 & 0 & 0 \\
1 & 0 & 0 & 0
\end{array}\right] \\
\boldsymbol{B}_{14}=-\boldsymbol{B}_{41}=\boldsymbol{B}_{23}=-\boldsymbol{B}_{32}=\left[\begin{array}{cccc}
0 & 0 & 0 & 1 \\
0 & 0 & 1 & 0 \\
0 & -1 & 0 & 0 \\
-1 & 0 & 0 & 0
\end{array}\right]
\end{gathered}
$$

$\boldsymbol{B}_{34}=\boldsymbol{B}_{43}=-\boldsymbol{B}_{12}=-\boldsymbol{B}_{21}$

$\boldsymbol{B}_{11}=\boldsymbol{B}_{22}=\boldsymbol{B}_{33}=\boldsymbol{B}_{44}=\boldsymbol{I}$

Denote $B=\left\{\left\langle\boldsymbol{B}_{s}, \boldsymbol{B}_{t}\right\rangle \mid s, t=1,2,3,4\right\}$ as a set, where $\left\langle\boldsymbol{B}_{s}, \boldsymbol{B}_{t}\right\rangle$ is the ordered couple relationship via the unitary transform of $\boldsymbol{B}_{s t}$ from $\boldsymbol{B}_{s}$ to $\boldsymbol{B}_{t}$. For Eqs. (26-30), $r, s, t \in\{1,2,3,4\}, \quad \boldsymbol{B}_{s t} * \boldsymbol{B}_{s t}^{\dagger}=\boldsymbol{I} ; \boldsymbol{B}_{s s} \in B$; if $\boldsymbol{B}_{s t} \in B$, then $\boldsymbol{B}_{t s} \in B$; if $\boldsymbol{B}_{r s} \in B$ and $\boldsymbol{B}_{s t} \in B$, then $\boldsymbol{B}_{r t} \in B$.

On the basis of set theory, four Bell bases satisfy the rule of equivalent relations including self-return, symmetry and transitivity via mutual unitary transforms. Moreover, any of
Bell bases can be transformed unitarily for quantum states evolutions.

\subsection{Three Controlled QT Schemes via GBBs}

To test whether the generalized Bell bases can be used in QIP fields or not, three controlled teleportation schemes with these GBBs are constructed to demonstrate their functions as Fig. (2).

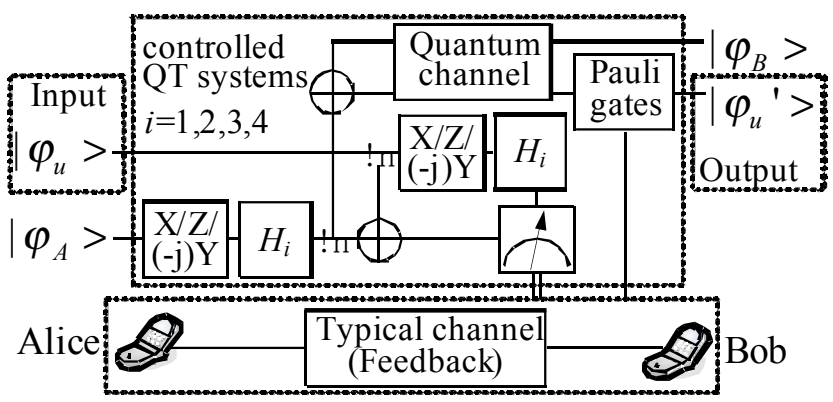

controlled object QT systems controller. X/Z/(-j)Y

Fig. (2). Controlled systems of QT schemes using GBBs.

Firstly, $\boldsymbol{B}_{1}$ is taken as an example to teleport an unknown state $\left|\varphi_{1}\right\rangle\left(\left|\varphi_{1}\right\rangle=a\left|0_{1}\right\rangle+b\left|1_{1}\right\rangle\right)$ from Alice (the sender) to Bob (the receiver) [1]. $a$ and $b$ are complex. $|a|^{2}+|b|^{2}=1$. The particles $2\left(\left|\varphi_{2}\right\rangle\right)$ and $3\left(\left|\varphi_{3}\right\rangle\right)$ from an EPR pairs $\left(\left|\beta_{11}^{B_{1}}\right\rangle_{23}\right)$ are shared by Alice and Bob. Using Bennett's method [1] in the first GBB-QT scheme, $\left|\varphi_{1}\right\rangle$ can be recovered and controlled by Bob via the typical channel with the feedback information of Bell bases from Alice:

$\left|\varphi_{123}\right\rangle=\sum_{i=00,01,10,11}\left|\beta_{i}^{B_{1}}\right\rangle_{12}\left|c_{i}\right\rangle_{3}$

Where $\left|\varphi_{123}\right\rangle$ is the tensor product of $\left|\varphi_{1}\right\rangle$ and $\left|\beta_{11}^{B_{1}}\right\rangle_{23}$. $\left|c_{i}\right\rangle_{3}$ can be solved respectively by the controllable Bell bases in Fig. (2) as follows:

$$
\begin{aligned}
& \left|c_{00}\right\rangle_{3}=\frac{1}{2} \mathrm{j} \boldsymbol{Y}\left|\varphi_{3}\right\rangle \\
& \left|c_{01}\right\rangle_{3}=\frac{1}{2}(-\boldsymbol{Z})\left|\varphi_{3}\right\rangle \\
& \left|c_{10}\right\rangle_{3}=\frac{1}{2} \boldsymbol{X}\left|\varphi_{3}\right\rangle \\
& \left|c_{11}\right\rangle_{3}=\frac{1}{2} \boldsymbol{I}\left|\varphi_{3}\right\rangle
\end{aligned}
$$

where $\left|\varphi_{3}\right\rangle$ (i. e. $a\left|0_{3}\right\rangle+b\left|1_{3}\right\rangle$ ) is a replica [1] of $\left|\varphi_{1}\right\rangle$. Similarly, the mathematical operations show that if $\left|\beta_{00}^{\boldsymbol{B}_{1}}\right\rangle_{23}$, 
$\left|\beta_{01}^{\boldsymbol{B}_{1}}\right\rangle_{23}$ or $\left|\beta_{10}^{\boldsymbol{B}_{1}}\right\rangle_{23}$ is a substitute for $\left|\beta_{11}^{\boldsymbol{B}_{1}}\right\rangle_{23}$, then $\left|\varphi_{1}\right\rangle$ can also be teleported.

Secondly, it is proved by the detailed derivations that if $\boldsymbol{B}_{2}$ and $\boldsymbol{B}_{3}$ displaces $\boldsymbol{B}_{1}$ as the other two GBB-QT schemes, then Alice can teleport unknown quantum states to Bob as well as using $\boldsymbol{B}_{1}$. Altogether, under the controls of the gates $\boldsymbol{X}, \mathbf{Z}$ and (-j) $\boldsymbol{Y}$ in Fig. (2), three GBBs can be utilized in controlled-QT as well as the traditional Bell basis in spite of lacking tests of Bell's inequalities violation.

\section{RESULTS}

By Controlling $\boldsymbol{B}_{1}, \boldsymbol{B}_{2}$ and $\boldsymbol{B}_{3}$ in Fig. (2) separately, the results of inverse unitary transform for Bob to restore unknown quantum states via controlled-QT are shown from Tables 1-3.

Table 1. Bob's unitary transforms via the first Bell basis.

\begin{tabular}{|c|c|c|c|c|}
\hline & $\left|c_{00}\right\rangle_{3}$ & $\left|c_{01}\right\rangle_{3}$ & $\left|c_{10}\right\rangle_{3}$ & $\left|c_{11}\right\rangle_{3}$ \\
\hline \hline$\left|\beta_{00}\right\rangle$ & $\boldsymbol{I}$ & $-\boldsymbol{X}$ & $-\boldsymbol{Z}$ & $\mathrm{j} \boldsymbol{Y}$ \\
\hline$\left|\beta_{01}\right\rangle$ & $\boldsymbol{X}$ & $-\boldsymbol{I}$ & $-\mathrm{j} \boldsymbol{Y}$ & $\boldsymbol{Z}$ \\
\hline$\left|\beta_{10}\right\rangle$ & $-\boldsymbol{Z}$ & $-\mathrm{j} \boldsymbol{Y}$ & $\boldsymbol{I}$ & $\boldsymbol{X}$ \\
\hline$\left|\beta_{11}\right\rangle$ & $-\mathrm{j} \boldsymbol{Y}$ & $-\boldsymbol{Z}$ & $\boldsymbol{X}$ & $\boldsymbol{I}$ \\
\hline
\end{tabular}

Table 2. Bob's unitary transforms via the second Bell basis..

\begin{tabular}{|c|c|c|c|c|}
\hline & $\left|c_{00}\right\rangle_{3}$ & $\left|c_{01}\right\rangle_{3}$ & $\left|c_{10}\right\rangle_{3}$ & $\left|c_{11}\right\rangle_{3}$ \\
\hline \hline$\left|\beta_{00}\right\rangle$ & $\boldsymbol{I}$ & $\boldsymbol{X}$ & $-\boldsymbol{Z}$ & $-\mathrm{j} \boldsymbol{Y}$ \\
\hline$\left|\beta_{01}\right\rangle$ & $\boldsymbol{X}$ & $\boldsymbol{I}$ & $-\mathrm{j} \boldsymbol{Y}$ & $-\boldsymbol{Z}$ \\
\hline$\left|\beta_{10}\right\rangle$ & $-\boldsymbol{Z}$ & $\mathrm{j} \boldsymbol{Y}$ & $\boldsymbol{I}$ & $-\boldsymbol{X}$ \\
\hline$\left|\beta_{11}\right\rangle$ & $-\mathrm{j} \boldsymbol{Y}$ & $\boldsymbol{Z}$ & $\boldsymbol{X}$ & $-\boldsymbol{I}$ \\
\hline
\end{tabular}

From Tables 1-4, no matter which Bell state is corresponding to the initial EPR pairs of Alice and Bob, unknown quantum states can be teleported and duplicated via inverse unitary transforms.

Through the selection of one or four Bell bases, the comparison results of the controlled QT-schemes via GBBs and the QT scheme using the typical Bell basis are shown as Table 5.
Table 3. Bob's unitary transforms via the third Bell basis.

\begin{tabular}{|c|c|c|c|c|}
\hline & $\left|c_{00}\right\rangle_{3}$ & $\left|c_{01}\right\rangle_{3}$ & $\left|c_{10}\right\rangle_{3}$ & $\left|c_{11}\right\rangle_{3}$ \\
\hline \hline$\left|\beta_{00}\right\rangle$ & $\boldsymbol{I}$ & $-\boldsymbol{X}$ & $\boldsymbol{Z}$ & $-\mathrm{j} \boldsymbol{Y}$ \\
\hline$\left|\beta_{01}\right\rangle$ & $\boldsymbol{X}$ & $-\boldsymbol{I}$ & $\mathrm{j} \boldsymbol{Y}$ & $-\boldsymbol{Z}$ \\
\hline$\left|\beta_{10}\right\rangle$ & $\boldsymbol{Z}$ & $\mathrm{j} \boldsymbol{Y}$ & $\boldsymbol{I}$ & $\boldsymbol{X}$ \\
\hline$\left|\beta_{11}\right\rangle$ & $\mathrm{j} \boldsymbol{Y}$ & $\boldsymbol{Z}$ & $\boldsymbol{X}$ & $\boldsymbol{I}$ \\
\hline
\end{tabular}

For comparisons, the quantum gates for Bob to restore unknown quantum states via QT with the traditional Bell basis [1] are collected in Table 4.

Table 4. Bob's unitary transforms via the fourth Bell basis.

\begin{tabular}{|c|c|c|c|c|}
\hline & $\left|c_{00}\right\rangle_{3}$ & $\left|c_{01}\right\rangle_{3}$ & $\left|c_{10}\right\rangle_{3}$ & $\left|c_{11}\right\rangle_{3}$ \\
\hline \hline$\left|\beta_{00}\right\rangle$ & $\boldsymbol{I}$ & $\boldsymbol{X}$ & $\boldsymbol{Z}$ & $\mathrm{j} \boldsymbol{Y}$ \\
\hline$\left|\beta_{01}\right\rangle$ & $\boldsymbol{X}$ & $\boldsymbol{I}$ & $\mathrm{j} \boldsymbol{Y}$ & $\boldsymbol{Z}$ \\
\hline$\left|\beta_{10}\right\rangle$ & $\boldsymbol{Z}$ & $-\mathrm{j} \boldsymbol{Y}$ & $\boldsymbol{I}$ & $-\boldsymbol{X}$ \\
\hline$\left|\beta_{11}\right\rangle$ & $\mathrm{j} \boldsymbol{Y}$ & $-\boldsymbol{Z}$ & $\boldsymbol{X}$ & $-\boldsymbol{I}$ \\
\hline
\end{tabular}

Table 5. Comparison results of QT schemes using GBBs and the typical Bell basis.

\begin{tabular}{|c|c|c|}
\hline & QT via the typical Bell basis & $\begin{array}{l}\text { Controlled-QT via } \\
\text { four GBBs }\end{array}$ \\
\hline 1 & One Hadamard gate & Four Hadamard gates \\
\hline 2 & Single Bell bases & Four Bell bases \\
\hline 3 & Limited measure basis & More measure bases \\
\hline 4 & Limited Bell states & Abundant Bell states \\
\hline 5 & Poor quantum information & Much quantum information \\
\hline 6 & $\begin{array}{c}\text { Less quantum entanglement } \\
\text { resource }\end{array}$ & $\begin{array}{c}\text { More quantum entanglement } \\
\text { resource }\end{array}$ \\
\hline 7 & $\begin{array}{l}\text { Only one kind of quantum } \\
\text { channel for QT }\end{array}$ & $\begin{array}{c}\text { Four kind of quantum channels } \\
\text { for QT }\end{array}$ \\
\hline 8 & $\begin{array}{l}\text { Fixed quantum circuit of Bell } \\
\text { basis }\end{array}$ & $\begin{array}{l}\text { Controllable quantum circuits } \\
\text { of Bell bases }\end{array}$ \\
\hline 9 & Sixteen QT schemes & Sixty-four QT schemes \\
\hline
\end{tabular}




\section{DISCUSSION}

Interestingly, Tables (1-4) are exceedingly similar with some laws, which illustrates that the QT schemes using these generalized forms of the traditional Bell basis [1] are of some reasonableness. One reasons is that $\boldsymbol{B}_{1}$ is equivalent to $\boldsymbol{B}_{3}$, while $\boldsymbol{B}_{2}$ is equivalent to $\boldsymbol{B}_{4}$ in the light of the superposition theorem of quantum state, and four Bell bases are of identical general expressions. The other reason is that the relations of unitary transformation among four Bell bases are equivalent, and these mutual equivalent relations result in the similar results of four quantum teleportation schemes.

Table 5 shows these controlled-QT schemes of GBBs have some advantages. Differently from the QT systems [1, 2], more quantum information of Bell state with the same general expression in Eq. (23) can be obtained than before via more Bell bases used in our QT schemes. The reason is that different outputs of Bell states with the same inputs are obtained by the unitary transforms of Pauli gates in Fig. (1). Two different groups of Bell bases result in the difference shown in Tables (1-4), where every item in one row of the initial Bell state is corresponding to a potential collapsed result of quantum entangled state for Bob during controlled GBB-QT. Using the controlled Pauli gates in Fig. (2), the collapsing results of quantum states vary with their different initial Bell states, and the changed quantum information of these results can be collected and utilized as new advantages of quantum resources in Tables 5.

\section{CONCLUSION}

By transforming unitarily the Hadamard gate with Pauli gates in the quantum circuit of the typical Bell basis, three new GBBs have been obtained, and the proposition that there are other similar Bell bases in four-dimensional Hilbert space is true. Four Bell bases have an identical general expression. Utilizing these GBBs, unknown quantum states can be teleported as well as using the traditional Bell basis. The controlled-QT schemes with GBBs have the advantages of simple unitary transforms and easy implementations by increasing three Pauli gates, which is the beneficial supplements of current GBS-QT work. From the GBB-QT processes, there exist some new available quantum entangled resources by the collapsed results of more Bell states, which can be used in such QIP fields as quantum key distribution, quantum entanglement swapping, quantum communication network, quantum repeater, etc. Also, the phase information of Bell bases needs to be exploited for QIP.

\section{CONFLICT OF INTEREST}

The authors confirm that this article content has no conflict of interest.

\section{ACKNOWLEDGEMENTS}

This work is supported by the National Natural Science Foundation of China (Nos. 61472094, 61371186) and the Guangxi Natural Science Foundation (No. 2013GXNSFF
A019004). We thank Prof. Nanrun Zhou for his helpful discussions.

\section{REFERENCES}

[1] C.H. Bennett, G. Brassard, C. Crépeau, R. Jozsa, A. Peres, and W.K. Wootters, "Teleporting an unknown quantum state via dual classical and Einstein-Podolsky-Rosen channels", Physical Review Letters, vol. 70, pp. 1895-1899, 1993.

[2] D. Bouwmeester, J.W. Pan, K. Mattle, M. Eibl, H. Weinfurter, and A. Zeilinger, "Experimental quantum teleportation", Nature, vol. 390, pp. 575-579, 1997.

[3] J. Yin, J.G. Ren, H. Lu, Y. Cao, H.L. Yong, Y.P. Wu, C. Liu, S.K. Liao, F. Zhou, Y. Jiang, X.D. Cai, P. Xu, G.S. Pan, J.J. Jia, Y.M. Huang, H. Yin, J.Y. Wang, Y.A. Chen, C.Z. Peng, and J.W. Pan, "Quantum teleportation and entanglement distribution over 100kilometre free-space channels", Nature, vol. 488, pp. 185-188, 2012.

[4] X.S. Ma, T. Herbst, T. Scheidl, D.Q. Wang, S. Kropatschek, W. Naylor, B. Wittmann, A. Mech, J. Kofler, E. Anisimova, V. Makarov, T. Jennewein, R. Ursin, and A. Zeilinger, "Quantum teleportation over 143 kilometres using active feed-forward", Nature, vol. 489, pp. 269-273, 2012.

[5] Y.F. Huang, X.F. Ren, Y.S. Zhang, L.M. Duan, and G.C. Guo, "Experimental teleportation of a quantum controlled-NOT gate", Physical Review Letters, vol. 93, p. 240501, 2004.

[6] S. Olmschenk, D.N. Matsukevich, P. Maunz, D. Hayes, L.M. Duan, and C. Monroe, "Quantum teleportation between distant matter qubits", Science, vol. 323, pp. 486-489, 2009.

[7] X.M. Jin, J.G. Ren, B. Yang, Z.H. Yi, F. Zhou, X.F. Xu, S.K. Wang, D. Yang, Y. F. Hu, S. Jiang, T. Yang, H. Yin, K. Chen, C. Z. Peng, and J.W. Pan, "Experimental free-space quantum teleportation", Nature, vol. 4, pp. 376-381, 2010.

[8] N.R. Zhou, L.J. Wang, L.H. Gong, X.W. Zuo, and Y. Liu, "Quantum deterministic key distribution protocols based on teleportation and entanglement swapping", Optics communications, vol. 284, pp. 4836-4842, 2011.

[9] M.X. Fu, F. Zhao, Y.Q. Lu, and S.H. Liu, "Progress of practical quantum key distribution network", Laser \& Optoelectronics Progress, vol. 44, pp. 39-47, 2007.

[10] C.X. Pei, Y. Yan, D. Liu, B.B. Han, and N. Zhao, "A quantum repeater communication system based on entanglement", Acta Photonica Sinica, vol. 37, pp. 2422-2426, 2008.

[11] N.R. Zhou, H.L. Cheng, L.H. Gong, and C.S. Li, "Three-party quantum network communication protocols based on quantum teleportation", International Journal of Theoretical Physics, vol. 53, pp. 1387-1403, 2014.

[12] L.Y. Hu, X.X. Xu, and S.J. Ma, “Application of generalized EPR entangled state in quantum teleportation", International Journal of Theoretical Physics, vol. 49, pp. 2486-2498, 2010.

[13] L. Vaidman, "Teleportation of quantum states", Physical Review A, vol. 49, pp. 1473-1476, 1994.

[14] J.J. Shao, X.L. Tian, and G.F. Shi, "Teleportation of N-qudit state", International Journal of Quantum Information, vol. 10, p. $1250013,2012$.

[15] H.W. Li, L.R. Long, P. Zhou, and C.L. Yin, "Quantum controlled teleportation of an arbitrary m-particle state using a $(2 m+1)$-particle entangled state as quantum channel", Acta Photonica Sinica, vol. 41, pp. 330-334, 2012.

[16] C. Lu, W. Zhang, H. Liu, L. Jia, and X.L. Tian, "Ququart State Teleportation", Acta Photonica Sinica, vol. 41, pp. 1394-1399, 2012.

[17] Y.B. Zhan, "Controlled teleportation of high-dimension quantumstates with generalized Bell-state measurement", Chinese Physics, vol. 19, pp. 2557-2562, 2007.

[18] G. Rigolin, "Quantum teleportation of an arbitrary two-qubit state and its relation to multipartite entanglement", Physical Review A, vol. 71, p. 032303, 2005.

[19] H.J. Cao, Z.H. Chen, Y.Y. Zhu, and H.S. Song, "Teleportation of a bipartite quantum state via generalized Bell states", Journal of Dalian University of Technology, vol. 50, pp. 469-474, 2010.

[20] P. Agrawal, and A.K. Pati, "Probabilistic quantum teleportation", Physics Letters A, vol. 305, pp. 12-17, 2002. 
[21] G. Gordon, and G. Rigolin, "Generalized teleportation protocol", Physical Review A, vol. 73, p. 042309, 2006.

[22] Y. Xia, J. Song, and H.S. Song, "Re-examining generalized teleportation protocol", Optics Communications, vol. 279, pp. 395398, 2007

[23] F.L. Yan, and T. Yan, "Probabilistic teleportation via a nonmaximally entangled GHZ state", Chinese Science Bulletin, vol. 55, pp. 902-906, 2010.

[24] Y.L. Liu, Z. X. Man, and Y.J. Xia, "Quantum teleportation of an arbitrary $N$-qubit state via non-maximally entangled state",
International Journal of Quantum Information, vol. 5, pp. 673-683, 2007.

[25] Y. Tanaka, M. Asano, and M. Ohya, "Quantum teleportation for nonmaximal entangled states in the generalized Bell measurement with Latin square", Reports on Mathematical Physics, vol. 69, pp. 57-74, 2012

[26] L.H. Gong, Y. Liu, and N.R. Zhou, "Novel Quantum Virtual Private Network Scheme for PON via Quantum Secure Direct Communication", International Journal of Theoretical Physics, vol. 52, pp. 3260-3268, 2013.

(C) Wu et al.; Licensee Bentham Open.

This is an open access article licensed under the terms of the Creative Commons Attribution Non-Commercial License (http://creativecommons.org/licenses/by-nc/3.0/) which permits unrestricted, non-commercial use, distribution and reproduction in any medium, provided the work is properly cited. 